\title{
Development of an integrated microfluidic platform for dynamic oxygen sensing and delivery in a flowing medium
}

\author{
Adam P. Vollmer, Ronald F. Probstein, Richard Gilbert and Todd Thorsen* \\ Received 8th June 2005, Accepted 16th August 2005 \\ First published as an Advance Article on the web 25th August 2005 \\ DOI: 10.1039/b508097e
}

This paper describes a platform for real-time sensing of dissolved oxygen in a flowing microfluidic environment using an oxygen-sensitive luminescent dye (platinum octaethylporphyrin ketone) integrated into a micro-oxygenator device. Using a phase-based detection method, the luminescent decay lifetime of the dye was consistent with the linear Stern-Volmer relationship using both gaseous and aqueous samples. Maximum sensor resolution varied between 120 $780 \mathrm{ppb}$ across a range of dissolved oxygen (DO) concentrations ranging from $0-42.5 \mathrm{ppm}$. The sensor was subsequently used to determine the convective mass-transfer characteristics of a multilayer polydimethylsiloxane (PDMS) microfluidic oxygenator. The membrane-based oxygenator showed excellent agreement with an analytical convection model, and the integrated oxygen sensor was accurate across a wide range of tested flow rates $\left(0.05-5 \mathrm{~mL} \mathrm{~min}^{-1}\right)$. The device is unique for its ease of fabrication and highly flexible configuration, as well as the novel incorporation of oxygen delivery and detection in a single micro-device. Potential applications include tissue engineering, cell culturing, and miniaturized bio-assays that require the delivery and/or detection of precise quantities of oxygen within a microfluidic construct.

\section{Introduction}

The delivery of a controlled oxygen supply and the accurate sensing of dissolved oxygen levels within a miniaturized environment is a necessity in biological assay, bioreactor, and tissue engineering applications. In cellular assays and bioreactors, rapid determination of cell viability is frequently accomplished by the monitoring of cellular metabolic activity via oxygen sensing. ${ }^{1,2}$ A controllable source of oxygen is a key constraint on cellular growth and development in tissue engineering applications. Newly implanted cells will consume the available oxygen supply within several hours, while it takes several days for angiogenesis to initiate blood vessel proliferation to provide growing tissue with oxygen and nutrients. ${ }^{3}$ As integrated microfluidic devices for such biological assay, reactor, and tissue engineering applications become increasingly ubiquitous, there is a compelling need for the development of flexible, low-cost oxygen sensors that can be rapidly prototyped and fabricated.

Many existing protocols for biochemical sensing in medical applications rely on intermittent sample extraction and external analysis in a blood-gas machine. This is a time consuming and inefficient process, ill-suited to the small sample volumes predominant in microfluidic and Micro Total Analysis Systems ( $\mu$ TAS). In addition to the difficulty of including sample ports within a micro-device, the concentration of oxygen gas in the analyte is an inherently unstable parameter, and sampling is thus likely to introduce error and measurement bias. For these reasons, integrated, real-time

Department of Mechanical Engineering. Massachusetts Institute of Technology, 77 Massachusetts Ave, Cambridge, MA 02139, USA. E-mail: thorsen@mit.edu measurement is desirable. The two predominant sensing techniques for chemical species detection in miniaturized biological applications are amperometric electrochemical sensing and luminescent optical sensing, both of which exhibit high sensitivity to low oxygen concentrations. ${ }^{4}$ Amperometric devices typically manifest as external Clark-type electrode sensors, although integrated devices have been demonstrated. ${ }^{1,5}$ However, for micro-device applications involving biological fluids and $\mu \mathrm{L} \mathrm{min}{ }^{-1}$ flow rates, amperometric sensors are generally undesirable because of difficulty in miniaturization, susceptibility to membrane fouling by organic matter, significant drift, analyte depletion, and signal variability at low flow rates. Alternatively, sensors based on optical excitation and detection of an embedded oxygen-quenched luminescent dye offer fast, linear response and immunity to drift from consumption of $\mathrm{O}_{2}$. These ideal characteristics have generated intense interest in luminescent chemical sensors, which have been demonstrated and marketed in a variety of configurations for various biological, environmental, aerospace, and other applications. ${ }^{2,6-15}$

With the specific intent of meeting the challenge of low-cost and ease-of-fabrication gas sensors, we report on the development of a luminescent oxygen sensor incorporated into a microfluidic oxygenation platform. Optical oxygen detection is achieved through the excitation of a luminescent dye suspended in a microporous polymer matrix. Polymer encapsulated oxygen-quenched lumophore sensors have previously been demonstrated with numerous luminescent dyes and substrates. Early work in the area focused on polyaromatic hydrocarbon complexes, ${ }^{16}$ with more recent work employing ruthenium diimines, ${ }^{14,17}$ and $\mathrm{Pt}$ and $\mathrm{Pd}$ porphyrin complexes. $^{2,6,9,10}$ Quantification of dissolved oxygen content using the dyes is determined by measurement of either the degree of 
luminescent intensity quenching or the luminescent lifetime. Of the previously mentioned dyes, the transition-metal $(\mathrm{Pd}, \mathrm{Pt})$ porphyrin complexes are most compatible with inexpensive solid-state optical components (LED and Si photodiodes). Detection can be performed either directly with fixed optics above and below the device ${ }^{18}$ or remotely with a fiber optic probe. $^{9,10}$ Both techniques allow non-invasive, real-time monitoring. In the present work, the former approach was employed to take advantage of the optical clarity of the polydimethylsiloxane (PDMS) microfluidic device and achieve a higher signal-to-noise ratio through better light coupling.

The fabrication process chosen for the microfluidic element was multilayer soft lithography, a technique well documented for "rapid prototyping" applications in easily variable configurations. ${ }^{19,20}$ The microfluidic oxygenator was constructed as a two-layer structure consisting of stacked parallel microchannels, with the bottom fluidic microchannels sealed to a glass slide. The lower, fluidic channel was fabricated in the design of a micro-capillary bed, while the upper channel allows controllable mass flow rates of oxygen gas in micro-channels oriented parallel to the fluidic channels. Diffusion between the two layers occurs readily across a thin silicone membrane separating them, due to the high permeability of PDMS to oxygen and other gases. Optical sensing pads were deposited at the inlet and outlet of the oxygen-transfer portion of the device. By monitoring the inlet and outlet gas concentration at various flow rates, the mass transfer characteristics of the device were characterized as a function of an effective dimensionless oxygenator "length". While previous work has demonstrated optical sensing of gaseous oxygen", ${ }^{9,11}$ and sensing of static fluids in biological microenvironments, ${ }^{7,8,12}$ the application of an integrated optical sensor for detection of dissolved oxygen in a flowing medium with active mass transfer is a unique development. A micro-device capable of oxygen sensing and delivery provides the potential for closedloop control and regulation of biochemical species, a particularly important and unique development for the types of biological micro-device and tissue engineering applications previously reported in literature. . $^{3,21}$

\section{Methods}

\section{Fabrication of SU-8 mold}

The molds for the gas and fluidic layers of the microfluidic devices were fabricated from SU-8 negative photoresist (Microchem) on a 3" $\mathrm{Si}$ wafer, producing patterned microchannel structures for subsequent casting with a height of $100 \mu \mathrm{m}$. Photolithography was performed using a high resolution transparency mask. Following development of the photoresist, the molds were silanized with a high molecular weight trichloro-perfluorooctyl silane (Aldrich) at room temperature for two hours to increases mold lifetime by promoting PDMS release from the Si/SU-8 surface. ${ }^{19,22}$

\section{Fabrication of multi-layer PDMS microfluidic element}

PDMS (General Electric RTV615) was chosen for the microfluidic devices based on its low cost, non-toxicity, and ease of fabrication with soft-lithography rapid prototyping techniques. PDMS is particularly appropriate for the oxygenator application because of its high permeability to oxygen gas. The diffusivity of oxygen in PDMS is approximately $6 \times 10^{-9} \mathrm{~m}^{2} \mathrm{~s}^{-1} \cdot{ }^{23}$ To construct the 2-layer microfluidic device, a 15 : 1 monomer/hardener mixture was mixed and degassed, then applied via spin-coating at a thickness of $120 \mu \mathrm{m}$ onto an SU-8 mold containing the fluidic channels. Simultaneously, a 5: 1 PDMS mixture was prepared and poured to a thickness of $\sim 6 \mathrm{~mm}$ onto a second SU-8 mold containing the gas channels. Prior to baking, both the PDMS spin-coated and poured molds were degassed under vacuum in a bell jar to remove air bubbles. The molds were then baked in an oven for $20 \mathrm{~min}$ at $80{ }^{\circ} \mathrm{C}$.

Following the initial bake, both molds were removed from the oven for alignment. The thick PDMS gas microchannel layer was cut and peeled from the SU- 8 mold and inlets were punched with fluidic vias using a blunt-tipped 20G surgical steel Luer stub. After punching, the bottom surface of the thick layer was washed with isopropyl alcohol to remove debris, aligned and mated to the spin-coated lower layer, and baked for an additional $2 \mathrm{~h}$ at $80{ }^{\circ} \mathrm{C}$ to covalently bond the layers together. After this final bake, the composite device was cut from the wafer, and the fluidic layer inlets were punched. Fig. 1 shows various images of the micro-oxygenator geometry, indicating the location of the sensor pads and the oxygenation section. The upper, gas-flow layer is a set of 64 densely packed parallel channels that perfectly overlays the oxygenator section in the fluidic layer. Examination of a device cross-section under a microscope confirms the accuracy of channel dimensions and vertical alignment (Fig. 1.c).

\section{Preparation of lumophore sensor}

Of the various available chemical dyes suitable for oxygen detection, Pt octaethyl-porphyrin-ketone (PtOEPK, Frontier Scientific) was selected for its desirable optical properties and compatibility with readily available and inexpensive solid-state electronic components. PtOEPK exhibits an absorption peak in the visible spectrum at $590 \mathrm{~nm}$, making it compatible with high-intensity yellow LED's. ${ }^{9}$ The emission peak of PtOEPK is $760 \mathrm{~nm}$, detectable by Si photodiodes. The longwave shift of the PtOEPK dye, relative to other luminescent materials, permits effective optical filtration with standard broadband filters. Additionally, PtOEPK shows greater long-term photostability than previously developed dyes. ${ }^{6}$ Oxygen permeable films of PtOEPK-polystyrene were prepared by dissolving $1 \mathrm{mg}$ of the dye in $1 \mathrm{~mL}$ of a $5 \%$ solution of polystyrene in toluene. A standard microscope slide was prepared as a substrate for the sensor using photolithography techniques. Positive photoresist (AZ 4620, Clariant) was spun to a thickness of $10 \mu \mathrm{m}$ and developed to leave two rectangular patches of glass exposed at the desired sensor locations on the glass slide. A commercially available dilute $\mathrm{HCl}$ glass etch (Armour Etch) was used to remove several microns of the glass surface, creating a microporous texture well suited to promoting mechanical adhesion between the glass substrate and the polystyrene matrix. A micropipette was used to apply 1-2 $\mu \mathrm{L}$ of the dye solution to the etched glass slide. After $10 \mathrm{~min}$ the solvent evaporated and acetone was used to strip the cured AZ 
(a)
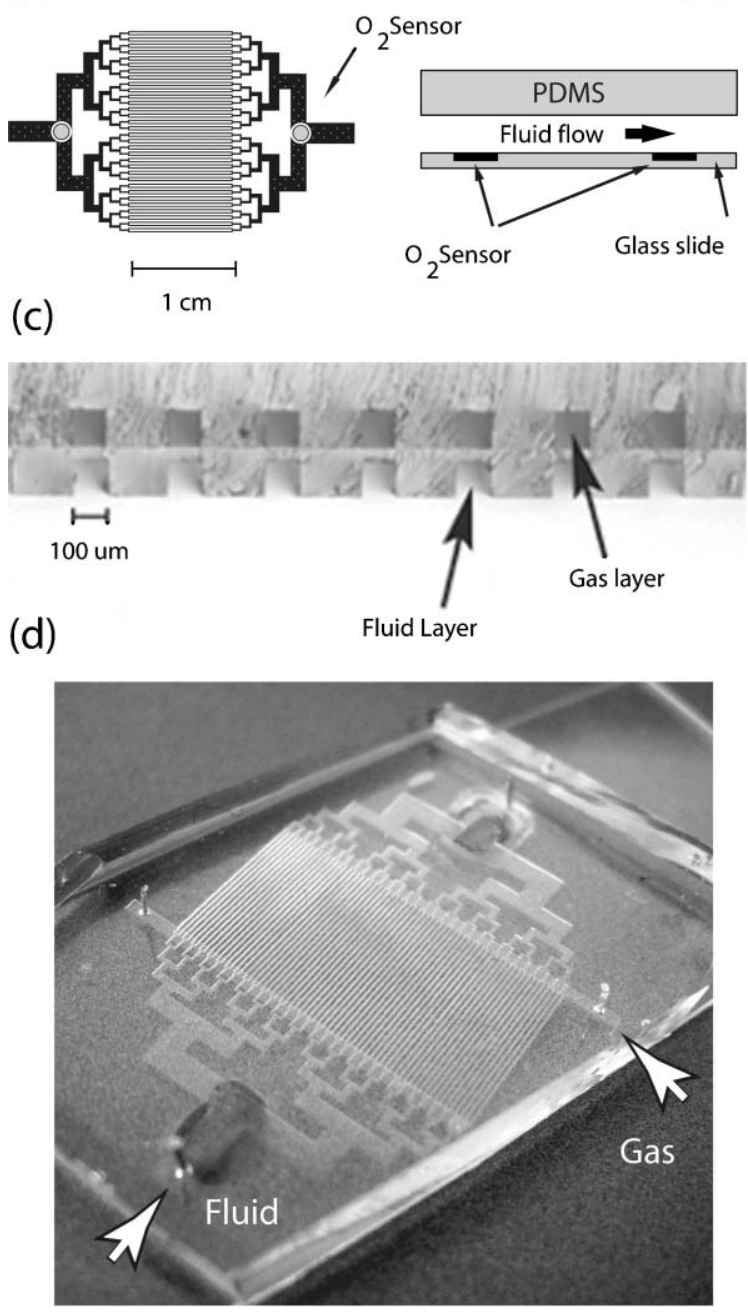

Fig. 1 (a) Lithography mask of capillary structure, showing location of $\mathrm{O}_{2}$ sensors. (b) Microfluidic device cross section, showing position of PtOEPK dye sensor pads etched in glass substrate. (c) Cross section view showing vertical alignment of fluidic and gas channels. Channels are separated by a $20 \mu \mathrm{m}$ membrane created by spin coating. (d) Image showing location of the sensor pads on an actual device, as well as the inlet locations for fluid and gas flow (arrows).

mask from the glass slide, leaving a $\sim 7 \mu \mathrm{m}$ thick polystyrene/ PtOEPK film. Acetone had a negligible effect on the integrity of the polystyrene-immersed dye.

After deposition of the sensors, oxygen-plasma bonding was used to seal the PDMS chip to the glass slide. Completed microfluidic structures were stored in the dark to prevent photobleaching and degradation of the sensor's optical properties. A fully assembled device is shown in Fig. 1.d.

\section{Optical and electrical apparatus}

An LED-based optical excitation and detection system was fabricated to monitor oxygen content in the microfluidic chip upstream and downstream of the oxygenator channels. A $15^{\circ}$ narrow-angle yellow/amber LED (590 nm, $2500 \mathrm{mcd}$, Ledtronic) was filtered by a BG39 Schott glass filter (CVI Laser) and positioned vertically directly above the sensor pad.
A Si photodiode (S2386-44k, Hamamatsu) was positioned below the sensor, shielded, and filtered by an RG715 Schott glass long-pass filter. Analog electronics amplified and filtered the photodiode current signal, which was recorded in real-time along with the reference LED signal on a PC via a USB DAQ card (PMD-1608FS, Measurement Computing). The excitation LED was modulated at $5 \mathrm{kHz}$, which allowed for phasedetection and low-frequency filtering in the signal conditioning circuitry to subtract any DC interference signal resulting from ambient lighting.

The luminescent lifetime of the PtOEPK dye, which is on the order of $60 \mu \mathrm{s},{ }^{9}$ was determined indirectly by measuring the phase shift between the excitation and emission waveforms. The relationship between phase shift, $\Theta$, and lifetime, $\tau$, in two common-frequency signals can be approximated by:

$$
\tan (\Theta)=2 \pi \nu \tau
$$

where $v$ is the modulation frequency. Phase-based lifetime detection precludes the need for high-speed $(\sim 10 \mathrm{MHz})$ data acquisition hardware to directly measure the luminescent decay time. For the $5 \mathrm{kHz}$ excitation and emission signals, phase shift was measured by digital lock-in detection in Labview. Additional post-processing was performed in Labview, which sampled the signal conditioning board at $20 \mathrm{kHz}$ and performed various filtering of recorded data to produce averaged data points at a rate of $5 \mathrm{~Hz}$. Lower averaged sampling rates $(<1 \mathrm{~Hz})$ produced less noise, but limited the dynamic response time of the system. The overall signal to noise ratio is maximized by the compact, vertical arrangement of the sensing components, shown in Fig. 2.

\section{Experimental procedure}

Fluidic and gas connections to the microfluidic device were made with $20 \mathrm{G}$ steel tube segments connected to Tygon tubing (Cole-Parmer). A syringe pump (Harvard Pump 11, Harvard Apparatus) was used to deliver a constant flow rate to the device. Reservoirs of deionized (DI) water were bubbled with

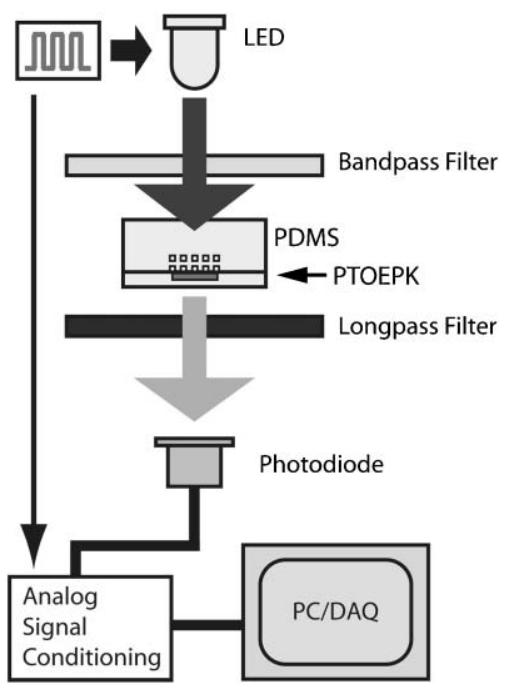

Fig. 2 Optical and electrical components and alignment in excitationdetection system. 
$\mathrm{N}_{2}$ and $\mathrm{O}_{2}$ gas for a minimum of $10 \mathrm{~min}$, providing sufficient time for the oxygen concentration to stabilize and provide $0 \%$ and $100 \%$ oxygen references. The DO concentration in oxygen-saturated water was calculated to be approximately $42.5 \mathrm{ppm}$, equivalent to $42.5 \mathrm{mg} \mathrm{L}^{-1}$ in a dilute aqueous solution at STP. Oxygen level in the reservoirs was continuously monitored by Clarke microelectrode sensors (DO-166FT, Lazar Research). Calibration tests of luminescent intensity and phase versus oxygen concentration were performed by perfusing the device with pure solutions of oxygenated and deoxygenated water and calibrated mixtures in ratios of $1: 3,2: 2,3: 1$ respectively. Additionally, the response of the PtOEPK film to purely gaseous samples was tested by blowing pure $\mathrm{O}_{2}, \mathrm{~N}_{2}$, and air over the sensor.

To test oxygenator performance, deoxygenated water was flowed through the system while the oxygen gas supply to the device's upper channels was cycled on and off, allowing the luminescent signal to equilibrate following each gas cycling. Fluid volume flow rate was varied from $50 \mu \mathrm{L} \min ^{-1}$ $5 \mathrm{~mL} \min ^{-1}$ during oxygenator testing, while properties of the channel geometry and membrane thickness were held constant. The flow rates of $50 \mu \mathrm{L} \mathrm{min}{ }^{-1}-5 \mathrm{~mL} \mathrm{m^{-1 }}$ represent flow rates through the entire 64 channel device, corresponding to flow rates of $0.78 \mu \mathrm{L} \mathrm{min} \operatorname{mos}^{-1}-78 \mu \mathrm{L} \mathrm{min}^{-1}$ through individual channels in the oxygenator section. Unless explicitly stated, flow rates in this paper will refer to aggregate rates through the entire microfluidic device.

\section{Results and discussion}

\section{Calibration and sensor performance in gaseous samples}

The oxygen quenching process occurring in PtOEPK and other luminescent dyes is described by the Stern-Volmer equation for luminescent intensity, $I$, and lifetime, $\tau$ :

$$
\begin{aligned}
& I_{0} / I=1+K_{\mathrm{Sv}}^{\mathrm{S}}\left[\mathrm{O}_{2}\right]=1+K_{\mathrm{Sv}}^{\mathrm{G}} p \mathrm{O}_{2} \\
& \tau_{0} / \tau=1+K_{\mathrm{Sv}}^{\mathrm{S}}\left[\mathrm{O}_{2}\right]=1+K^{\mathrm{G}}{ }_{\mathrm{Sv}} p \mathrm{O}_{2}
\end{aligned}
$$

where $K_{\text {SV }}^{\mathrm{S}}$ and $K^{\mathrm{G}}{ }_{\mathrm{SV}}$ are the Stern-Volmer constants for solution and gas, respectively, $I_{0}$ and $\tau_{0}$ are reference values in the absence of oxygen, $\left[\mathrm{O}_{2}\right]$ is the oxygen concentration in solution, and $p \mathrm{O}_{2}$ is the gaseous partial pressure of oxygen. Luminescent lifetime decay values were determined from the phase shift, as described by eqn (1), and luminescent intensity was determined from the amplitude of the emitted $5 \mathrm{kHz}$ waveform. Phase-based detection of luminescent lifetime was selected as the preferred detection scheme for all calibration and experimental measurements. A significant advantage of phase-based lifetime measurements is the inherent stability of the signal. The luminescent lifetime is an intrinsic property of the PtOEPK molecule and is therefore not susceptible to variation based on the intensity of incident light or heterogeneity in the thickness or distribution of the dye-polystyrene matrix. In contrast, the magnitude of the emitted luminescent intensity varies in proportion to the magnitude of incident light, meaning that small changes in alignment of the sensor pad can result in significant measurement errors. For this reason, the phase-based lifetime measurement is a considerably more reliable determinant of oxygen concentration than the luminescent intensity.

Sensor calibration was initially performed using gaseous mixtures. Fig. 3.a shows the dynamic response of the sensor to pure gas flow, and Fig. 3.b shows the corresponding 3-point Stern-Volmer curve. Oxygen partial pressure is normalized in Fig. 3.b between 0 and 1, corresponding to pure nitrogen and oxygen, respectively. Fig. 3.a demonstrates high reversibility and nearly instantaneous dynamic response of the sensor under gas flow. The total time for $90 \%$ sensor equilibration is on the order of one second. Sensor linearity between normalized lifetime and oxygen concentration is excellent in detection of gaseous samples, as shown in Fig. 3.b. Whereas non-linearity has been observed in many applications of polymer-suspended dye sensors, ${ }^{11}$ the high diffusivity of oxygen in the hydrophobic polystyrene matrix improves the sensitivity of this sensor and contributes to its linearity across the entire operating range of oxygen concentration.

\section{Calibration and sensor performance in fluid samples}

Detection of dissolved oxygen in aqueous samples was tested using calibrated concentrations of gas in buffered DI water. Dynamic sensor response is slower in an aqueous testing environment, varying from $30 \mathrm{~s}$ at flow rates above $4 \mathrm{~mL} \mathrm{~min}^{-1}$ to $3-5 \mathrm{~min}$ at flow rates below $500 \mu \mathrm{L} \mathrm{min}{ }^{-1}$. There are two primary causes for the slower dynamic response of the sensor in a flowing liquid medium. First, the interaction between dissolved oxygen (DO) and the luminescent dye is limited by the rate of DO diffusion through the fluid. Second, and most important for sensing across a broad dynamic range of flow
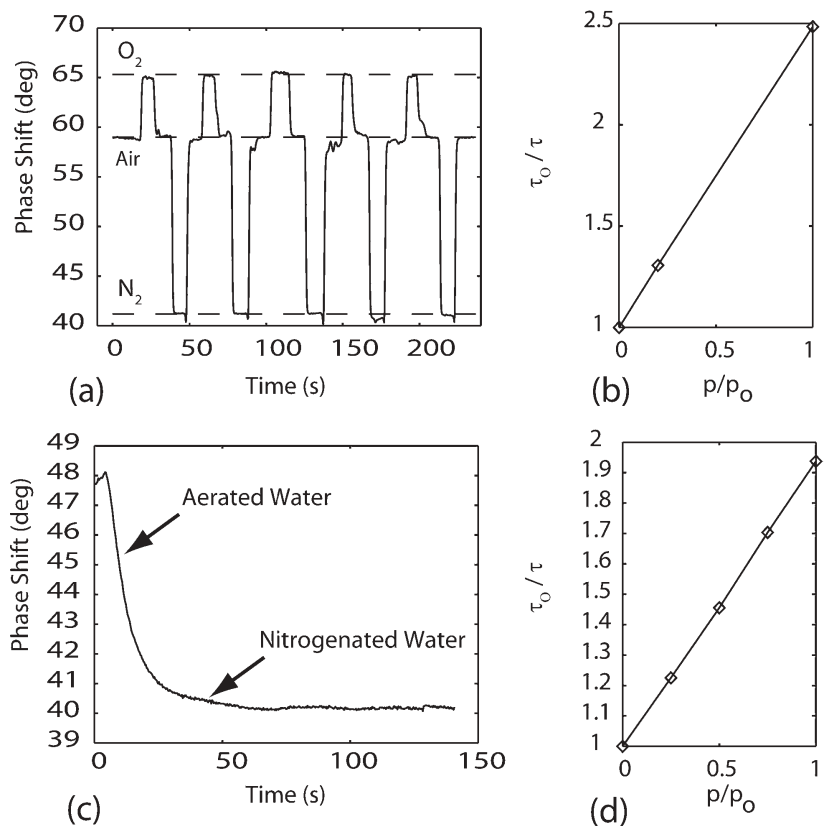

Fig. 3 (a) Dynamic sensor response (phase shift, $\Theta$ ) for detection of gaseous oxygen. (b) Stern-Volmer calibration curve for detection of gaseous oxygen, according to eqn (3). (c) Dynamic sensor response (phase shift, $\Theta$ ) for detection of dissolved oxygen, $Q=1 \mathrm{~mL} \mathrm{~min}{ }^{-1}$. (d) Stern-Volmer calibration curve for detection of dissolved oxygen, according to eqn (3), $Q=1 \mathrm{~mL} \mathrm{m^{-1 }}$ 
rates, is the equilibration that must occur between the local oxygen content in the PDMS and the oxygen content in the fluid. PDMS is highly permeable to oxygen - thus its selection as a membrane material for the oxygenator. A convective process occurs between the PDMS walls of the microchannel and the oxygen-bearing fluid. Depending on the concentration of oxygen in the fluid entering the device, mass transfer from the walls occurs to either add or remove DO from the fluid. Equilibration occurs once the PDMS walls in the vicinity of the sensor have reached an oxygen concentration equal to the fluid. This process occurs more rapidly with faster moving fluids, resulting in the longer settling times at low flow rates. Through various sensor designs and methods of integrating the dye, we have accomplished nearly a ten-fold increase in speed of response over the evolution of the device. Fig. 3.c and 3.d show the dynamic response of the sensor and Stern-Volmer plot, respectively, for detection of $\mathrm{DO}$ at a flow rate $(Q)$ of $1 \mathrm{~mL} \mathrm{m^{-1 }}$. Despite the slower dynamic response time for DO, sensor linearity remains excellent, as shown in Fig. 3.d.

To evaluate the effectiveness of the microfluidic oxygenator, we demonstrated the repeatability of sensor measurements across a broad dynamic range of flow rates. Fig. 4.a shows the phase shift data for nitrogenated and oxygenated water at flow rates between $50 \mu \mathrm{L} \mathrm{min}^{-1}$ and $5 \mathrm{~mL} \mathrm{~min}^{-1}$. At low flow rates ( $Q<2 \mathrm{~mL} \mathrm{~min}^{-1}$ ), the full-scale operating range of the sensor decreases due to parasitic convective losses to the surrounding PDMS. Phase shift measurements across the full range of tested flow rates were normalized between the oxygenated
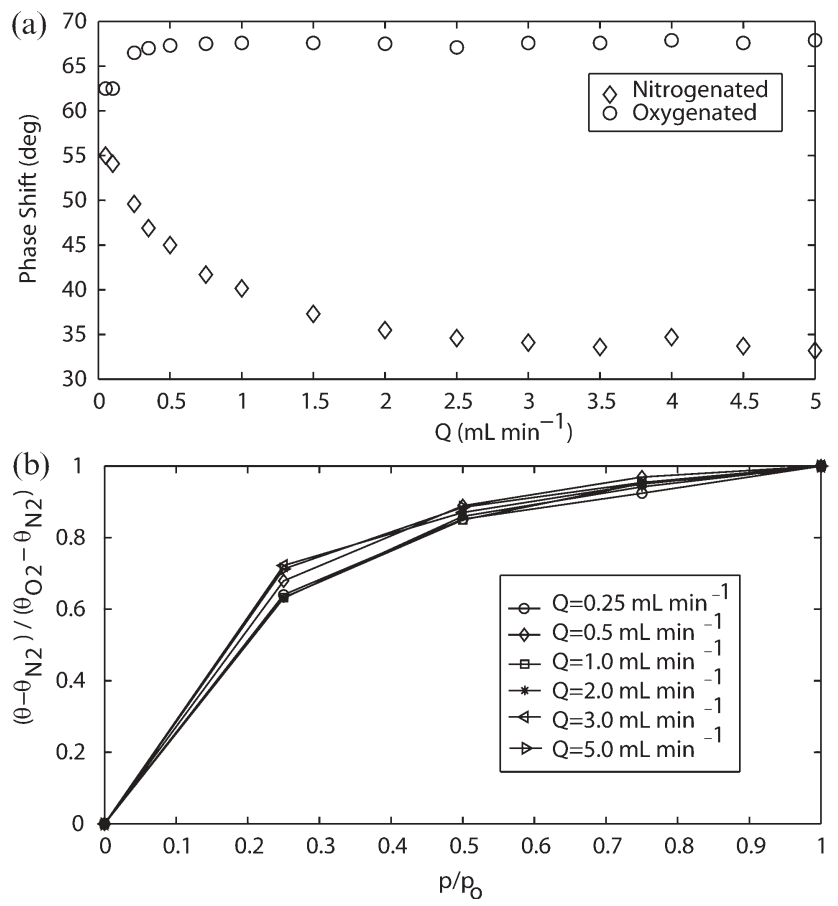

Fig. 4 (a) Phase shift data corresponding to oxygenated and deoxygenated water across entire dynamic operating range of flow rates. Convective losses are exacerbated at low flow rates, requiring normalization of data between upper and lower bounds, Fig. 4.b. (b) Calibration curves of normalized phase shift versus oxygen ratio for flow rates between $0.05-5 \mathrm{~mL} \mathrm{~min}^{-1}$. Normalized data demonstrates the sensor's repeatability at various flow rates.
$(100 \%)$ and deoxygenated $(0 \%)$ phase shift, according to the following formula:

$$
\Theta_{\mathrm{NORM}}=\frac{\Theta_{\text {MEASURED }}-\Theta_{\mathrm{N}_{2}}}{\Theta_{\mathrm{O}_{2}}-\Theta_{\mathrm{N}_{2}}}
$$

where $\Theta_{\mathrm{N}_{2}}$ and $\Theta_{\mathrm{O}_{2}}$ are the measured values, for a particular flow rate, with deoxygenated and oxygenated water. Fig. 4.b shows the normalized 5-point calibration for 6 different flow rates. The collapse of the normalized phase shift data to a single point at $y=0$ (nitrogenated water) and $y=1$ (oxygenated water) reflects the lower and upper bounds of eqn (4). This 5-point calibration was performed at all flow rates to achieve maximum accuracy in subsequent experimental work.

Resolution for the detection of dissolved oxygen varies in proportion to the flow rate. At $50 \mu \mathrm{L} \mathrm{min}{ }^{-1}$, the resolution is $780 \mathrm{ppb}$, or $0.92 \%$ of the full-scale oxygen concentration. At flow rates above $3 \mathrm{~mL} \mathrm{~min}^{-1}$, the sensor approaches a maximum resolution of $120 \mathrm{ppb}, 0.14 \%$ oxygen. The resolution and accuracy of the sensor for detection of both gaseous samples and fluidic samples compares favorably with results previously demonstrated. ${ }^{11,12}$ The range and resolution of the sensor make it ideally suited to detection of dissolved oxygen in biological fluids such as blood.

\section{Photobleaching and stability}

A common concern with lumophore-based chemical sensors is the degradation of the sensor's quantum efficiency following prolonged sampling and continual illumination. To evaluate the sensor's optical stability, a PtOEPK-polystyrene film was exposed to ambient air $\left(20.1 \% \mathrm{O}_{2}\right)$ and data was recorded over a period of $3 \mathrm{~h}$, pulsing the LED at $20 \mathrm{kHz}$, corresponding to nearly constant illumination. As shown in Fig. 5, the intensitybased signal demonstrated a slight deterioration over the three hour course of the test. The change in observed voltage was $8 \mathrm{mV}$, corresponding to an error of $2.75 \%$ over the full-scale range of the sensor.
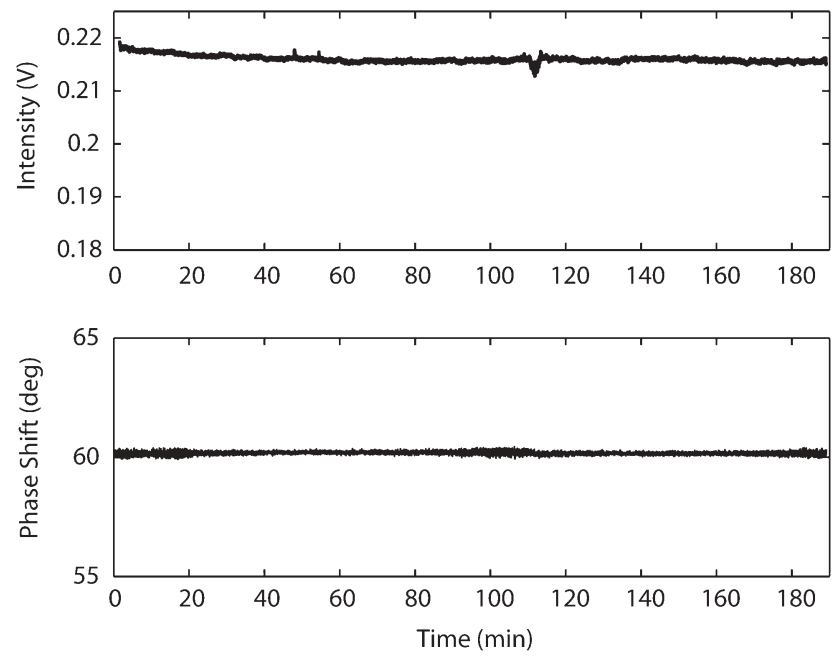

Fig. 5 Photobleaching test showing stability of sensor in phasebased regime. The intensity-based signal demonstrates minor photo-degradation. 
The phase-based measurement of the luminescent lifetime was minimally affected by photobleaching effects over the three hour test period, justifying the selection of phase-based lifetime as the preferred sensing technique. Based on the lower limit on response time in liquid, approximately $30 \mathrm{~s}$, the sampling rate of the measurement apparatus could be reduced from $5 \mathrm{~Hz}$ to $0.2 \mathrm{~Hz}$, sampling every $5 \mathrm{~s}$, with a negligible loss in dynamic resolution. Reducing the sampling rate by a factor of 25 would deliver the same dose of illumination to the device as produced in our photobleaching test, over a period of approximately $72 \mathrm{~h}$ rather than $3 \mathrm{~h}$. This places the operational lifetime of the sensor on the order of at least 3 days, suitable for any number of biological applications involving the growth or monitoring of cells in a micro-environment over a time span of several days.

\section{Oxygenator characterization and sensor application}

The mass transfer properties of the microfluidic oxygenator were characterized analytically by developing a simple convective mass transfer model, modelling the square channels of the actual device as infinite parallel plates. Experimental results obtained using the integrated PtOEPK sensor were compared to this model to determine the validity of the analytical work and demonstrate the model's appropriateness for simulations of future device designs. The parallel channel geometry of a single channel in the oxygenator is shown in Fig. 6. Oxygen diffusion in the two-layer microchannel oxygenator occurs from the $\mathrm{O}_{2}$ gas reservoir in the upper channel through the PDMS membrane into the adjacent fluid channel. As shown in Fig. 6, mass transfer begins at the point $x=0$ and continues along the length of the channel to the end of the channel, where $x=\mathrm{L}$. The hydrodynamic entry length is on the order of 1.5-150 $\mu \mathrm{m}$, depending on the flow rate, which is a variable in the experiment. Relative to the $1 \mathrm{~cm}$ overall length of the channel, the extremely short entry length justifies the assumption of a fully developed hydrodynamic profile in the model.

The ratio of the length of the developing region for the concentration boundary layer to the channel height is approximated by the Peclet number,

$$
P e=\frac{U H}{D_{\mathrm{O}_{2}}}
$$

where $U$ is the mean fluid velocity, $D_{\mathrm{O}_{2}}$ is the diffusivity of oxygen in the fluid, and $H$ is the channel height (see Fig. 6). The Peclet number is a measure of the ratio of the convective to diffusive mass transport. The individual channel Peclet numbers are in the range of 50-5000, indicating that, except in the range of the lowest of the experimentally evaluated flow rates $\left(Q=0.8 \mu \mathrm{L} \min ^{-1}\right.$, per channel), mass transfer occurs in the form of a developing concentration boundary layer. The developing concentration boundary layer, as well as the fully developed concentration profile, are shown in Fig. 6. Defining a nondimensional "effective channel length", $L^{*}$, in eqn (6), it can be shown that the developing concentration boundary layer becomes fully developed at approximately $\mathrm{L}^{*} \sim 1$.

$$
L^{*}=\frac{L}{H} \frac{1}{P e}
$$

This result will be important in the calculation of mass transfer coefficients in the different regimes of developing and developed flow.

Boundary and initial conditions for the diffusion problem are described as follows. The mass transfer coefficient between the $\mathrm{O}_{2}$ reservoir and inner wall of the membrane is several orders of magnitude higher than the mass transfer coefficient for diffusion into the moving fluid. Because of the extremely high transfer coefficient across the membrane, the diffusive resistance of the membrane is effectively ignored, and the boundary condition at the inner membrane surface $\left(C_{y=0}\right)$ is modelled as constant at the saturated concentration $C_{\mathrm{SAT}}$, where $C$ is the concentration of dissolved oxygen, measured in $\mathrm{mg} \mathrm{L}^{-1}$. The remaining, non-membrane contacting walls of the fluidic channel (the bottom channel wall in the model) are taken to be impermeable to oxygen. The normal concentration gradient at these walls is therefore zero. Fluid enters the oxygenation portion of the channel at the deoxygenated freestream concentration of $C_{x=0}=C_{\mathrm{O}}$.

The steady-state convective-diffusion equation for binary diffusion in dilute liquid solutions relates the concentration of the diffusing species $\left(\mathrm{O}_{2}\right)$ to the axial, $x$, and longitudinal, $y$, coordinates of the channel as follows:

$$
u_{x} \frac{\partial C}{\partial x}-D_{\mathrm{O}_{2}} \frac{\partial^{2} C}{\partial y^{2}}=0
$$

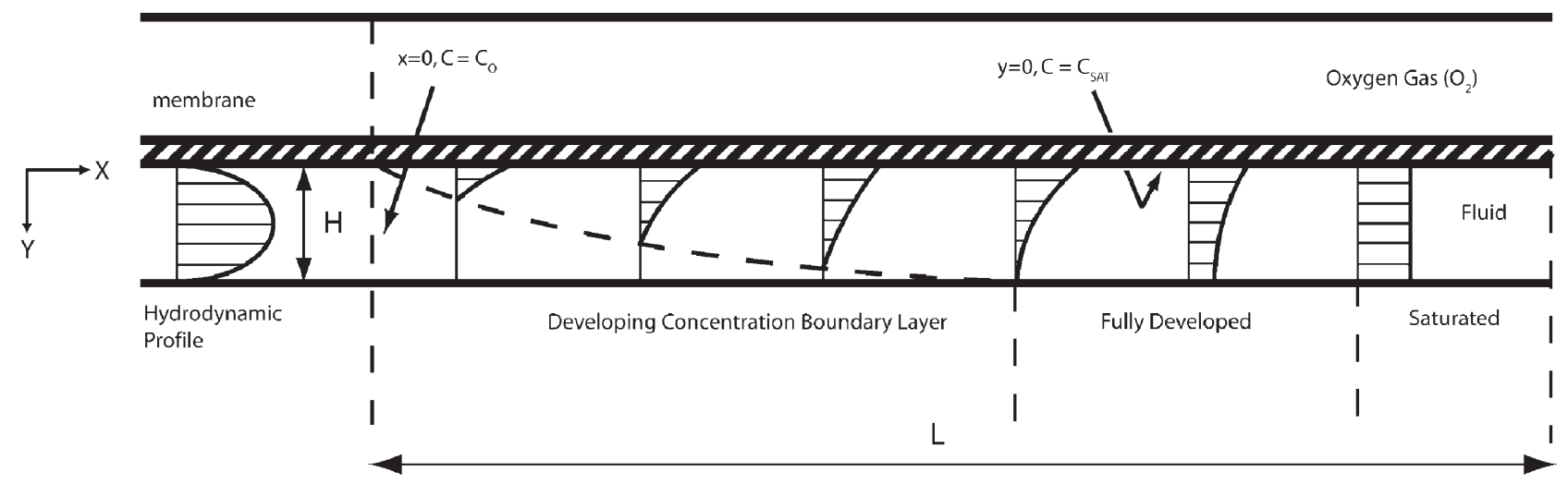

Fig. 6 Geometry of the mass transfer scenario for the microfluidic oxygenator, detailing the parallel plate geometry, parabolic fluid velocity, $\mathrm{O}_{2}$ reservoir, membrane, and the regimes of developing, fully developed, and saturated gas concentration profiles. 
Here, $u_{x}$ is the fluid velocity in the axial direction. Eqn (7) states that the axial convection of a gaseous species is balanced by the transverse diffusion of the species.

The following equations are defined to facilitate a solution to eqn (7). At the inner surface of the membrane, the diffusive mass flux of oxygen is defined per unit time per unit area as follows:

$$
j=D_{\mathrm{O}_{2}} \frac{\partial C}{\partial y}=h_{D}\left(C_{\mathrm{SAT}}-C_{\mathrm{M}}\right)
$$

where $h_{D}$ is the mean mass transfer coefficient. The value $C_{\mathrm{M}}$ is the "mixed mean", or bulk average, concentration, at any cross section. The mixed mean concentration $C_{\mathrm{M}}$ quantifies an 'average' oxygen concentration at an axial position in the channel and is defined by:

$$
C_{\mathrm{M}}(x)=\frac{\int C(x, y) \times u_{x}(y) \mathrm{d} A}{\int u_{x}(y) \mathrm{d} A}
$$

Employing the definition of mixed-mean concentration $C_{\mathrm{M}}$ and wall flux $j$ (eqn (8)), the diffusion equation (eqn (7)) can be integrated across the channel cross-section, assuming a constant mean mass transfer coefficient, yielding the following relation:

$$
\frac{\left(C_{\mathrm{SAT}}-C_{\mathrm{M}}(L)\right)}{\left(C_{\mathrm{SAT}}-C_{\mathrm{O}}\right)}=\exp \left[\frac{-L}{H U} h_{D}\right]
$$

Eqn (10) describes the relation between the normalized mean oxygen concentration and the mean mass transfer coefficient, channel length $L$, and mean fluid velocity $U$.

Evaluation of eqn (10) requires an appropriate mean mass transfer coefficient, $h_{D}$. The mass transfer coefficient is determined from the Sherwood number, $S h$, a dimensionless parameter which measures the ratio of the mass transfer flux to the diffusive driving force.

$$
S h=\frac{j H}{\left(C_{\mathrm{SAT}}-C_{\mathrm{M}}\right) D_{\mathrm{O}_{2}}}=\frac{h_{D} H}{D_{\mathrm{O}_{2}}}
$$

Here, $\left(C_{\mathrm{SAT}}-C_{\mathrm{M}}\right)$ characterizes the diffusive driving force, the difference between the saturated concentration at the membrane wall of the channel and the mixed-mean concentration in the fluid. The Sherwood number can be computed for both the regions of developing and fully developed oxygen concentration in a variety of channel cross sections. For simplicity, the square channel geometry has been modelled as a hydrodynamically fully developed flow between infinite parallel plates, with an impermeable boundary condition on one wall and a constant concentration on the opposing wall.

The experimental flow rates under consideration vary across several orders of magnitude, meaning that the diffusive concentration profile may be either developing or fullydeveloped, depending on the value of $L^{*}$ (eqn (6)). In the developing boundary layer region, exact computations of the Sherwood number rely on a similarity solution of eqn (7) that utilizes a linearization of the parabolic velocity profile near the membrane wall known as the Leveque approximation. ${ }^{24}$ Such an approximation is only valid for boundary layers which are extremely thin relative to the channel width. Therefore, the analytical solution for the developing boundary layer concentration profile is exact only for values of $L^{*}$ considerably less than 1, for which the boundary layer thickness is thin. For values of $L^{*}$ on the order of 1 or greater, the concentration profile is fully developed and the Sherwood number is constant. ${ }^{25}$ Based on these two solutions, a piecewise function for the Sherwood number can be defined that is valid over approximate ranges of $L^{*}$ (eqn (6)) given below:

$$
\begin{gathered}
S h=1.233 L^{*-1 / 3} \text { for } L^{*} \ll 1(\text { ref. } 24) \\
S h=4.861 \text { for } L^{*} \geqslant 1(\text { ref. } 25)
\end{gathered}
$$

Eqn (12) is plotted in Fig. 7, where the solutions in both ranges of $L^{*}$ are extrapolated into the transition regime where an exact solution is not available. From Fig. 7, the thin-boundary-layer Leveque solution intercepts the fully developed solution at approximately $L^{*}=0.02$. As a simple approximation the extrapolated solutions are taken to hold up to their intersection, that is,

$$
\begin{gathered}
S h=1.233 L^{*-1 / 3} \text { for } L^{*}<0.02 \\
S h=4.861 \text { for } L^{*} \geqslant 0.02
\end{gathered}
$$

Applying the definition of the Sherwood number given in eqn (13) to the solution of eqn (10) and comparing to the results of analogous experimental heat transfer data given in Shah and London, ${ }^{25}$ it was verified that the approximate form of $S h$ in eqn (13) yields a maximum error of less than $5 \%$ in determining the mean concentration.

Fig. 8 shows the parallel plate model result given by eqns (10) and (13), together with the experimental data obtained for the percentage oxygen concentration delivered by the oxygenator at a particular effective length, $L^{*}$. The data is plotted on a semi-logarithmic scale with percent oxygenation defined relative to the calibration endpoints, where $0 \%$ represents nitrogen-bubbled water and $100 \%$ represents oxygen-bubbled water $\left(0-42.5 \mathrm{mg} \mathrm{L}^{-1}\right)$. Note that, since Fig. 8 displays percent oxygen concentration relative to the inlet concentration, the quantities derived from eqns (10) and (13) for a single channel are equally valid for the entire 64

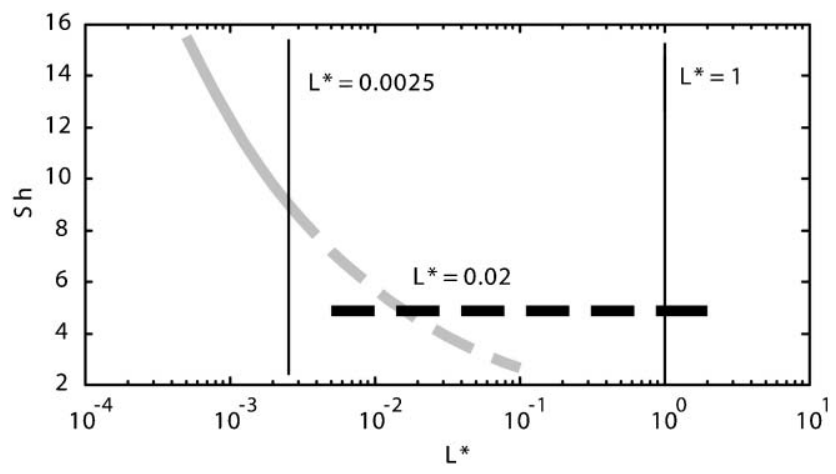

Fig. 7 Sherwood number $(S h)$ plotted versus effective length $\left(L^{*}\right)$. For the transition regime between $0.0025<L^{*}<1$, the concentration boundary layer Leveque solution (grey) and the fully developed solution (black) have been extrapolated beyond their formal range of applicability. The Leveque solution intersects the fully developed solution at $L^{*} \approx 0.02$. In the present analysis, $S h$ is approximated as a piecewise function defined by the Leveque solution below $L^{*}=0.02$ and the fully developed solution for $L^{*}>0.02$. This approximation was verified to produce minimal error. 


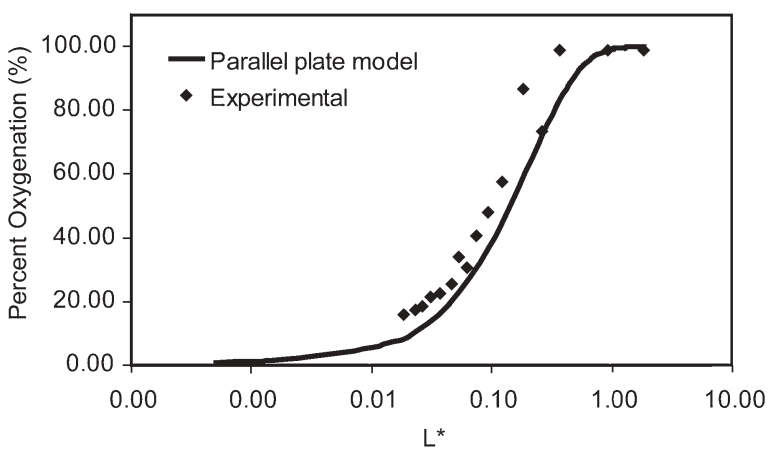

Fig. 8 Experimental and analytical results for the mixed-mean $\mathrm{O}_{2}$ concentration plotted versus $L^{*}$.

channel oxygenator network. Fig. 8 shows that the experimental data is in good agreement with the simple convective mass transfer model of eqns (10) and (13). It is apparent that the infinite parallel plate model utilized for the mass transfer analysis is an approximation to the actual square geometry. We expect that the true mass transfer coefficient for a square channel will be slightly higher than the value presented in eqn (13), due to corner effects in channels with finite aspect ratios. In future iterations of microchannel and microoxygenator design, the good agreement between experiment and theory obtained here supports the use of the simple convectivediffusion model to predict device characteristics and optimize device geometry. For a given device in a particular application, the parameter $L^{*}$ may be altered in real-time via the flow rate to deliver a precise and repeatable concentration of oxygen between $0-100 \%$ saturation in water, on-demand.

\section{Conclusions}

This paper describes the development of a unique integrated microfluidic platform for precise delivery and detection of gaseous or dissolved oxygen. Multilayer soft lithography was used to create a PDMS capillary network suitable for diffusing oxygen into a moving fluid, tested at flow rates from $50 \mu \mathrm{L} \min ^{-1}$ to $5 \mu \mathrm{L} \mathrm{min}{ }^{-1}$. Real-time oxygen sensing in the flowing microchannels was accomplished using integrated luminescent oxygen sensors deposited on the capillary substrate. The sensors were evaluated using both gaseous and aqueous samples, verifying their linearity, optical stability, resolution, and accuracy. The microfabricated sensors have a maximum resolution of $120 \mathrm{ppb}$, and excellent long-term stability in the phase-sensing regime. Experimental microchannel oxygenation characteristics were accurately predicted by a simple convective mass transfer model. The optical sensor performance (sensitivity, resolution) is comparable to recently published sensors of a similar nature, ${ }^{8,11,12}$ with a simpler fabrication protocol and a more flexible platform for detection of gaseous or dissolved oxygen. The compactness, demonstrated biocompatibility of the PDMS material, ${ }^{26}$ and rapid, inexpensive fabrication capability of the device make it ideally suited to a range of biological applications in various configurations. Foreseeable applications include tissue engineering and cell culturing in a microfluidic environment, real-time blood gas detection in a clinical environment, or extracorporeal oxygenation in a surgical setting. Future areas of investigation will involve testing of biological fluids, such as blood, in the device, in order to optimize channel geometry and material characteristics. Additionally, we aim to investigate the question of scaling, to increase the volume flow rate capacity of the device and accommodate near-physiological fluid flow rates while maintaining the individual microchannel properties and highly efficient gas transfer characteristics of the current module. This work highlights the potential for integrating proven sensor technology into flexible microdevice configurations for a wide range of applications ideally suited to $\mu$ TAS and Lab-On-A-Chip style devices.

\section{Acknowledgements}

We would like to gratefully acknowledge Harry Lee for our many enlightening conversations. This work has been partially supported by the National Institutes of Health (NIH) under grant number DC 05604.

\section{References}

1 M. Brischwein, E. R. Motrescu, E. Cabala, A. M. Otto, H. Grothe and B. Wolfe, Lab Chip, 2003, 3, 234-240.

2 T. C. O'Riordan, D. Buckley, V. Ogurtsov, R. O'Connor and D. B. Papkovsky, Anal. Biochem., 2000, 278, 221-227.

3 H. Andersson and A. V. d. Berg, Lab Chip, 2004, 98-103.

4 R. Ramamoorthy, P. K. Dutta and S. A. Akbar, J. Mater. Sci., 2003, 38, 4271-4282.

5 S. M. Mitrovski and R. G. Nuzzo, Lab Chip, 2005, 5, 634-645.

6 D. B. Papkovsky, Sens. Actuators, B, 1995, 29, 213-218.

7 A. Zanzotto, N. Szita, P. Boccazzi, P. Lessard, A. J. Sinskey and K. F. Jensen, Biotechnol. Bioeng., 2004, 87, 243-254.

8 Y. F. Cao, Y. E. L. Koo and R. Kopelman, Analyst, 2004, 129, 745-750.

9 D. B. Papkovsky, G. V. Ponomarev and W. T. a. P. Oleary, Anal. Chem., 1995, 67, 4112-4117.

10 D. B. Papkovsky, A. N. Ovchinnikov, V. I. Ogurtsov, G. V. Ponomarev and T. Korpela, Sens. Actuators, B, 1998, 51, 137-145.

11 C. S. Burke, O. McGaughey, J. M. Sabattie, H. Barry, A. K. McEvoy, C. McDonagh and B. D. MacCraith, Analyst, 2005, 130, 41-45.

12 D. A. Chang-Yen and B. K. Gale, Lab Chip, 2003, 3, 297-301.

13 E. R. C. a. J. N. Demas, B. A. DeGraff and J. R. Bacon, Anal. Chem., 1991, 63, 337-342.

14 D. P. O’Neal, M. A. Meledeo, J. R. Davis, B. L. Ibey, V. A. Gant, M. V. Pishko and G. L. Cote, Sens. J. IEEE, 2004, 4, 728-734.

15 W. Trettnak, C. Kolle, F. Reininger, C. Dolezal and P. O'Leary, Sens. Actuators, B, 1996, 36, 506-512.

16 I. Bergman, Nature, 1968, 218, 396.

17 I. Klimant and O. S. Wolfbeis, Anal. Chem., 1995, 67, 3160-3166.

18 W. Trettnak, C. Kolle, F. Reininger, C. Dolezal, P. O’Leary and R. A. Binot, Adv. Space Res., 1998, 22, 1465-1474.

19 G. M. Whitesides, E. Ostuni, S. Takayama, X. Y. Jiang and D. E. Ingber, Annu. Rev. Biomed. Eng., 2001, 3, 335-373.

20 T. Thorsen, S. J. Maerkl and S. R. Quake, Science, 2002, 298, $580-584$.

21 J. T. Borenstein, H. Terai, K. R. King, E. J. Weinberg, M. R. Kaazempur-Mofrad and J. P. Vacanti, Biomed. Microdev., 2002, 4, 167-175.

22 J. B. Brzoska, I. Benazouz and F. Rondelez, Langmuir, 1994, 10, 4367-4373.

23 J. Brandrup and E. H. Immergut, in Polymer Handbook, 2nd edn, 1974.

24 R. F. Probstein, in Physicochemical Hydrodynamics, WileyInterscience, Hoboken, NJ, 2nd edn, paperback, 2003.

25 R. Shah and A. London, in Laminar Flow Forced Convection in Ducts: a Source Book for Compact Heat Exchanger Analytical Data, Academic Press, New York, 1978.

26 M. C. Belanger and Y. Marois, J. Biomed. Mater. Res., 2001, 58, 467-477. 